lerdings ist die Grenze der Polarwüste festgelegt durch die Temperatur; im Grenzsaum aber hat die Temperatur kein Primat, vielmehr entspricht die Kältewüste weitgehend einer Trockenwüste.

Staub, Walther, Bern: Über die «praeglaciale 》Verebnung im Quellgebiet von Rhone, Reuß und Rhein. - Die Längstäler der Rhone, der Urseren-Reuß, des Vorderrheins, wie das Bedrettotal eignen sich auch deswegen für morphologische Feststellungen, weil hier auf große Längenerstreckungen Steil- und Senkrechtstellung der Gesteinsfolgen vorherrscht, alte Abtragungsflächen und Terrassen daher leicht als solche erkannt werden können. Hier werden seit langem die Verebnungen, welche an der oberen Waldgrenze zwischen rund 2000-2200 m Meereshöhe auftreten, als «praeglacialer» Talboden angesprochen. In diesen sind die glazialen Trogtäler eingesenkt. Die als «praeglacial» angesprochene Fläche bildet im Hintergrund einiger Täler auch die oberste Stufe der heutigen Talsohle und läßt sich von hier talauswärts mit Unterbrüchen als Terrasse oder wenigstens Gehängeleiste verfolgen. Ohne Talstufe setzt sie sich in die Seitentäler fort. Oft ist diese Terrasse als «Boden» oder «Platte » in hochgelegene Talwurzeln zu verfolgen. Mindestens vom mittleren Wallis bis in das Davosergebiet ist diese Abtragungsfläche ein höchst wichtiger Boden für die Alpwirtschaft. Die Herausbildung muß sehr lange Zeit in Anspruch genommen haben; sie kann nur tertiären Alters sein und auf ihr spielte sich die älteste, greifbare Entwässerung ab. Bei $2300-2400 \mathrm{~m}$ Höhe wird diese Fläche meist von den Resten einer höheren, älteren begleitet, in welche nicht selten Karböden eingesenkt sind. In diesem Zustand der Alpen tritt die Furka als klare Wasserscheide zwischen West und Ost bereits hervor, während die Grimsel $(2164 \mathrm{~m})$, der Gotthard (2091 m) und der Oberalppaß (2044 m) in dieser alten Fläche liegen. Zunächst muß der Aarmassivrücken die Wasserscheide zwischen Nord und Süd gewesen sein. Mit Hilfe der Höhenschichtenkarte, welcher die neue topographische Darstellung zugrunde liegt, läßt sich nun zeigen, daß das Aaretal und die Schöllenen als jüngere Erosionstäler in diese alte Einebnungsfläche eingefallen, also jünger sind. Die glazialen Täler weisen eine auffallende Treppenform, sowohl in der Längsrichtung, wie im Querschnitt auf. Es scheint, daß im Alter das Goms dem Tavetsch gleichzustellen ist.

\title{
DER DERZEITIGE STAND \\ DER FORSCHUNG IN METEOROLOGIE UND KLIMATOLOGIE
}

\author{
MAX SCHÜEPP
}

Die Meteorologie hat in den letzten zwei Jahrzehnten durch die Entwicklung des Flugwesens eine starke Förderung erfahren, vor allem durch die damit verbundene Verdichtung des Beobachtungsnetzes auf den Ozeanen und in den höheren Luftschichten. Der Transatlantikverkehr, welcher seit dem zweiten Weltkrieg einsetzte, brachte die Errichtung von stationären Wetterschiffen, die zwar infolge der hohen Kosten von ursprünglich 13 auf 10 und neuerdings auf 9 reduziert wurden, aber immer noch das Grundgerippe für die Erforschung der wichtigen Wetterentwicklungen auf dem Ozean bilden, da sie täglich Radiosondenaufstiege in die freie Atmosphäre durchführen. Dabei wurde festgestellt, daß in mittleren Breiten in einer schmalen Zone, am häufigsten im Winterhalbjahr um $40^{\circ}$ Breite, ein Gebiet starker Höhenwinde existiert, der sogenannte jet-stream (Strahlstrom oder Düsenstrom). Dieser erreicht in der oberen Troposphäre, der Höhenlage der stärksten Winde, oft Geschwindigkeiten von $200-300 \mathrm{~km} / \mathrm{Std}$., in einzelnen Fällen sogar noch größere Werte. Durch die systematische Erforschung der Windverhältnisse in der Tropenzone, im Gebiet des Passatkreislaufes, durch Radiosondenmessungen bei allen Wetterlagen wurde ferner festgestellt, daß die in der klassischen Theorie der allgemeinen Zirkulation vorhandene Antipassatströmung kaum existiert, indem das Abströmen der Luft von der Äquatorregion nach den Subtropen nicht, wie theoretisch erwartet werden sollte, mit westlichen Windkomponenten erfolgt. Das Abströmen erfolgt sehr wahrscheinlich in einer korkzieherartigen Reibungszirkulation innerhalb des tropischen Ostwindgebietes. Diese Fest- 
stellungen führten in den letzten Jahren seit dem Krieg zu intensiveren Studien über die allgemeine Zirkulation, besonders durch den schwedischen Forscher C. G. RossBy und eine mit ihm arbeitende Forschergruppe in Chicago. Die Erklärung, welche sich heute aufdrängt, geht nicht mehr aus von der Passatzirkulation als treibendem Motor des ganzen Wettergeschehens, sondern vom Gegensatz der verschiedenen Luftmassen in den gemäßigten Breiten der Erde an der sogenannten Polarfront, an welcher sich Zonen sehr großen meridionalen Temperaturgefälles und als Folge davon die jetstream-Bänder entwickeln. Die kinetische Energie dieser langgezogenen Strömungsfelder wird in den großen Hoch- und Tiefdruckgebieten, welche die tägliche Wetterkarte zeigt, in Wirbelbewegungen und nachfolgend in Reibungswärme umgewandelt. Die Hoch- und Tiefdruckzentren wandern zwar mit der allgemeinen Strömungsrichtung von Westen nach Osten, doch sammeln sich die Tiefdruckgebiete infolge der im Norden und Süden ungleich großen ablenkenden Kraft der Erdrotation (Corioliskraft) vorwiegend nördlich der Polarfront, in der Zone um $60^{\circ}$ Breite, während die Hochdruckzellen in die $30^{\circ}$-Breitenzone, in die Subtropen abwandern. Die neuen Auffassungen, welche in der geographischen Literatur vor allem durch $\mathrm{H}$. FLOHN in den vergangenen Jahren dargelegt wurden, sind auch an einer Studientagung der schweizerischen Geographielehrer im geographischen Institut der Universität Zürich in diesem Sommer besprochen worden.

Naturgemäß bietet die Verarbeitung des gewaltigen, heute anfallenden meteorologischen Beobachtungsmaterials große Schwierigkeiten, die man in den meisten Staaten durch die Benutzung von Lochkarten zu vermindern sucht. Die bedeutende Aufgabe, das ganze Lehrgebäude allen festgestellten Tatsachen anzupassen, bleibt jedoch bestehen. Es ist somit nicht verwunderlich, wenn überall auf der Welt nach der fast völligen Abschnürung während der Kriegszeit in den letzten Jahren ein intensiver Gedankenaustausch eingesetzt hat. Die Konferenzen folgen sich in fast ununterbrochener Folge. So muß z. B. die technische Weiterentwicklung der Instrumente, speziell der Radiosonden, immer wieder besprochen werden, bei denen verschiedenartige Systeme mit leider auch etwas ungleichen Meßergebnissen mit einander in Konkurrenz stehen. Ferner waren in den Jahren nach dem letzten Krieg auch die internationalen Schlüssel (Codes) zur Übermittlung der Meldungen neu festzulegen, was bei den bestehenden Gegensätzen zwischen der westlichen und östlichen Welt manche Debatte an den Konferenzen der OMM (Organisation Météorologique Mondiale) verursachte. Da letzten Endes aber nur eine zirkumpolare Wetterkarte Antwort auf die zu lösenden Probleme geben kann, sind beide Teile gleich an einem Verständigungswerk interessiert, so daß der 1946 in Paris beschlossene und 1947 in Washington wieder etwas abgeänderte Code für die Wettermeldungen auf den 1. Januar 1955 in bereinigter Fassung vorliegt. Im Interesse der klimatologischen Auswertung ist nun zu wünschen, daß die weiteren Konferenzen keine neuen Änderungen bringen werden, da sonst speziell für die Lochkartenbearbeitung der Meldungen Schwierigkeiten entstehen.

Die Klimatologie ist zwar in weniger rascher Entwicklung als die synoptische Meteorologie begriffen, doch versucht auch sie zu neuen Bearbeitungsmethoden zu gelangen. Wie auf geographischem Gebiet der Landschaftsbegriff, d. h. die Unterteilung des vorliegenden Materials, eines der Hauptprobleme bildet, so stellt sich auch für den Klimatologen das Problem der Einteilung sowohl großräumig, als auch bei der Bearbeitung der einzelnen Regionen. Schon verschiedentlich ist der Versuch gemacht worden, die klassischen großräumigen Einteilungen, z. B. die Köppensche durch eine mit der allgemeinen Zirkulation in engerem Zusammenhang stehende dynamische Einteilung zu ersetzen. Bisher hat sich aber kein neues System durchsetzen können. Auch in der praktischen Bearbeitung der Beobachtungsergebnisse eines bestimmten Landes bestehen immer noch Unterschiede zwischen den einzelnen Staaten, z. B. in der Wahl der Bezugsperiode (1901 - 30, 1891 - 1930, $1901-40,1901-50,1921-50$, um nur einige der in Europa am meisten verwendeten Perioden zu nennen). An der Konferenz der klimatologischen Kommission der OMM in Washington im vergangenen Jahr wurde neben der bisher international empfohlenen Reduktionsperiode 1901-30 die Periode 1921-50 vorgeschlagen, welche in späterer Zeit laufend durch die Perioden 1931-60 usw. ersetzt werden soll, so daß eine allgemeine Regelung erzielt ist. Da aber im Gegensatz zu der Synoptik in der Klimatologie die einzelnen Länder weitgehend autonom sind und ein Wechsel in den Beobachtungs- und Auswertemethoden große Arbeit sowie eventuell den Verzicht auf homogene Beobachtungsreihen bringt, ist es sehr fraglich, ob schon in naher Zukunft Einheitlichkeit der Auswertungen erzielt werden kann.

Ein wichtiges Gebiet, welches die Verbindung zwischen Klimatologie und Synoptik herstellen soll, die Witterungsklimatologie, d. h. die Untersuchung bestimmter Wetterlagen statt der Verwendung von Monats- und Jahresmitteln der einzelnen Wetterelemente, ist erst in Entwicklung begriffen. Die Witterungsklimatologie dürfte aber dazu berufen sein, einen Fortschritt in der Wetterprognose zu bringen, indem die einzelnen Wetterabläufe genauer analysiert und ihre Ursachen erforscht werden. Wichtig ist zunächst die Aufstellung eines genauen Klassifikationsschemas. Für die großräumige Druckverteilung existiert eine solche Einteilung von F. BAUR in der Neubearbeitung von Hess-Brezowsky. An der in diesem Frühjahr in Davos durchgeführten Tagung für alpine Meteorologie wurde das Problem der Klassifikation im Alpenraum in mehreren Vorträgen aufgeworfen, und es soll bald von der Schweiz aus ein entsprechender Vorschlag herausgegeben werden. Im Alpengebiet stellen sich - wie das 66 Vorträge umfassende Davoserprogramm zeigte überhaupt die verschiedenartigsten Probleme in Bezug auf Schneeforschung, Strahlungsforschung, Bioklimatologie und Synoptik. Alle zwei Jahre erfolgt eine Zusammenkunft der speziell an alpiner 
Meteorologie interessierten Forscher zur Orientierung über den derzeitigen Stand der Arbeiten und die sich stellenden Probleme.

Ein weiteres Arbeitsgebiet betrifft die experimentelle Meteorologie, welche an einer ebenfalls internationalen Tagung in Zürich wom 4.-6. Oktober dieses Jahres behandelt wurde. Besonders in Amerika wird versucht, in den Trockengebieten durch künstliche Impfung der Wolken mit Silberjodid (Ag I) die Zahl der Kondensationskerne zu erhöhen und damit die Niederschlagsbildung zu fördern. Nach den Angaben des auf diesem Gebiet tätigen Referenten besteht ein gewisser Erfolg, indem, soweit die bisherigen statistischen Unterlagen beurteilen lassen, eine $10-20 \%$ ige Mehrausbeute in den bearbeiteten Regionen erzielt wurde. Daneben laufen Versuche zur Hagelbekämpfung mit ähnlichen Mitteln. Auch die Schweiz besitzt in der Magadinoebene ein Versuchsgebiet für die Erprobung der Hagelabwehr mit Raketen und den neuen Silberjodidzerstäubern. Es ist jedoch sehr schwer, aus kurzen Versuchsreihen von wenigen Jahren sichere Schlüsse über die Wirksamkeit zu ziehen. Daher wird neben den praktischen Versuchen besonders die theoretische Erforschung der Ursachen der Niederschlagsbildung genauer untersucht. Es handelt sich darum, die Unklarheit über die Tropfenbildung zu beseitigen, z. B. abzuklären, ob der Niederschlag vorwiegend durch Zusammenfließen (Koagulation) der kleinen Tröpfchen oder durch Ansatz an einfallenden Eiskriställchen erfolgt.

Ein weiteres speziell an der im September dieses Jahres in Rom durchgeführten internationalen Tagung der Union Géodésique et Géophysique Internationale (UGGI) behandeltes Thema betrifft die Synoptik: die numerische Vorhersage von Wetterkarten, d. h. der Luftdruckverteilung in Meeresniveau und in der $500 \mathrm{~m} / \mathrm{b}$ Fläche (ca. $5500 \mathrm{~m}$ Höhe). An Stelle der bisherigen empirischen Methoden der Vorhersage soll die exakte Berechnug mit Hilfe der Elektronen-Rechenmaschine, eventuell sogar mit einfacheren Hilfsmitteln treten. Die bisherigen, auf $24-72$ Stunden vorausberechneten Karten zeigen zwar in einzelnen Fällen noch wesentliche Abweichungen und sind zur Zeit kaum besser als die mit den bisherigen Methoden extrapolierten Karten, lassen aber doch die Hoffnung aufkommen, da $\beta$ in den kommenden Jahren und Jahrzehnten dieser Weg erfolgreich beschritten werden kann. Die Witterungsklimatologie muß dann allerdings zusätzlich Auskunft geben, wie das vorausberechnete Luftdruckbild sich wettermäßig in den Prognosenelementen Wind, Temperatur, Bewölkung und Niederschläge auswirken wird. Bis zur völlig objektiven Methode der Wettervorhersage wird daher noch ein weiter Weg zurückzulegen sein, den wir aber hinter uns bringen müssen, wenn auf dem Prognosengebiet in Zukunft wesentliche Fortschritte erzielt werden sollen.

In unserem Land werden die Wetterprognosen gegenwärtig und wohl auch noch einige Zeit nach den alten, auf den theoretischen Kenntnissen und der Erfahrung des diensttuenden Meteorologen beruhenden Vorhersagemethoden ausgegeben. Es wurde jedoch in den vergangenen Jahren versucht, durch eine bessere regionale Einteilung genauere Angaben über die einzelnen Klimagebiete zu geben. Auch soll zur besseren Orientierung das tägliche Wetterbulletin der Meteorologischen Zentralanstalt ab 1 . Januar 1955 etwas umgestaltet werden, indem der technische Teil - die codifizierten Wettermeldungen - in vergrößerter Form auf einem Separatblatt erscheinen wird und die Zahl der im Klartext meldenden Stationen des Bulletins vermehrt wird. Ferner sollen neben der großen Wetterkate des Raumes Europa-Atlantik von morgens $7 \mathrm{~h}$ die Höhenwetterkarte von morgens $4 \mathrm{~h}$ und die zugehörige Temperatur- und Windverteilung über der Aerologischen Station Payerne graphisch dargestellt werden. Hoffen wir, daß diese Neuerungen, welche eine vermehrte Berücksichtigung des Beobachtungsmaterials aus der freien Atmosphäre bringen, das Verständnis der Wetterabläufe innerhalb und außerhalb des meteorologischen und geographischen Fachkreises fördern werden.

\section{GESELLSCHAFTSTÄTIGKEIT - ACTIVITE DES SOCIETES}

Vortragstätigkeit. Basel. 22. Oktober 1954. Dr. T. A. Schinzel, Basel: Kunst und Religion in Indien; 5. November. O. Jenny-Lehner, Oberdorf: Pyrenäen und Baskenland; 12. November: Führung im Museum für Völkerkunde: Kunstwerke vom Sepik; 19. November. Prof. Dr. R. v. HeineGeldern, Wien: Chinesische und indische Einflüsse in der Kunst der alten Mexikaner und Maya; 3. Dezember: E. Rauch, dipl. Ing. agr.; Zürich: Vielgestaltiges Nepal; 17. Dezember. Prof. Dr. H. ВовEк, Wien: Klima und Landschaft Irans in vor- und frühgeschichtlicher Zeit. Bern. 5. September. Exkursion Bern - Wasen i. E. - Huttwil - Willisau - Sursee-Beromünster-Sempach-WertensteinWolhusen (Leitung PD. Dr. W. STAuB); 15. Oktober: Frau F. Hauser-TroxleR, Bern: Meine Reise nach Indien und Nepal; 29. Oktober. Dr. P. Amsler, Bern: Korea; 19. November. H. Zwicky: Dolomitenfahrt: 22. November. Prof. Dr. R. v. Heine-Geldern, Wien: Vorkolumbische Beziehungen zwischen Asien und Südamerika; 3. Dezember. Dr. W. Kündig-STEIner, Zürich: Vorderasien heute; 16. Dezember. Dr. R. WeRner, Zürich: Boghazköy und Van, Kulturzentren des Alten Orientes. St. Gallen. 21. August-17. Oktober. Stadtjubiläums-Ausstellung im Neuen Museum. 29. Oktober. Dr. J. Maeder: Am Rande der Sahara; 16. November. H. Sturzenegger, Wabern-Bern: Irland-Land unter Wolkenschatten; H. N. BıschoF, St. Gallen: Korea-Land und Leute. Ziirich. 3. November. Prof. Dr. H. Gutersohn, Zürich: Ceylon; 17. November: Prof. Dr. R. v. Heine-Geldern, Wien: Chi- 